\title{
(c)
}

(c) Auteur. Cette œuvre, disponible à

http://dx.doi.org/10.18162/fp.2021.a231, est distribuée

sous licence Creative Commons Attribution 4.0 International

http://creativecommons.org/licences/by/4.0/deed.fr

\section{7 clefs pour des interactions pédagogiques porteuses d'apprentissages}

doi: 10.18162/fp.2021.a231

Tantôt fécondes (Webb, 1983), tantôt malsaines (Perrenoud, 1998), les interactions pédagogiques ne sont ni nécessaires pour apprendre ni suffisantes (Perrenoud, 1987). L'objet de cette chronique, travail de synthèse, est de proposer sept conseils pour promouvoir de saines interactions porteuses d'apprentissages.

\section{Interactions en général}

\section{Centrez-vous sur les objectifs d'apprentissage}

Commençons par le conseil le plus ambivalent. D’un côté, l'interaction n'est formatrice qu'en tant qu'elle participe à l'éducation ou aux apprentissages, c'est-à-dire quand elle pousse au partage de connaissances, à une construction commune, à la compétition, à la coordination de points de vue, à une coopération intellectuelle (Perrenoud, 1987). De l'autre, certaines activités débordent sainement des objectifs, parce que l'enseignant nourrit des intentions qui dépassent les comportements observables chez l'étudiant (Hameline, 1979). C'est que les objectifs, pour centraux qu'ils soient, ne mènent pas toujours aux effets escomptés. Ainsi, une activité interactive, en plus d'être alignée sur les objectifs et l'évaluation (Biggs, 1996), devrait éviter la seule acquisition d'informations ou la régurgitation des savoirs (Comeaux et al., 1998). 


\section{Obtenez I'adhésion et faites-vous plaisir}

La meilleure des activités ne fonctionnera pas si l'étudiant n'y adhère pas, soit parce qu'il n'en perçoit pas l'intérêt, soit parce qu'il ne perçoit aucune conviction chez l'enseignant. Prenons conscience du fait que «pour les étudiants, les priorités sont inversées par rapport à celles du professeur : l'examen est primordial et les cours sont accessoires» (Mazur, 2014, p.27). Est-ce à dire qu'un dialogue de sourds est inévitable? Assurément non, à condition de respecter trois ingrédients : des attentes claires, des normes partagées et un climat positif.

L'expression des attentes concerne les exigences, les critères de réussites, les délais. Être explicite réduit les résistances. Une activité ayant l'air facultative est rarement réalisée, alors que proposer des contraintes est foncièrement fécond (Korczak, 1920/2006; Houdé, 2017). Quant aux normes, souvent floues en enseignement à distance, elles doivent être réinventées (Mazur, 2014), alors quelles sont si claires pour un enfant de 10 ans qui sait exactement ce que l'on attend de lui. Faut-il allumer les caméras? Ditesle. Quel niveau de langage est attendu dans les chats et forums? Spécifiez-le. Faut-il lever la main pour prendre la parole? Exprimez-le. Enfin, parce que l'interaction peut être source de dénigrement, d'exclusion, et d'autres effets néfastes (Perrenoud, 1998), il est crucial de créer un climat social positif. Par exemple, en nommant chacun par son nom ou en ouvrant les salles virtuelles un peu avant et après les horaires du cours. Les moments de sains échanges, sous la condition d’être exempts de jugement, stimuleront la motivation et protégeront de l'abandon. Parler de ces bienfaits est utile afin que bavards et introvertis trouvent place dans une authentique interaction didactique (Perrenoud, 1998).

\section{Interactions asynchrones}

\section{Utilisez les forums}

Le forum est un espace virtuel à coloniser, un «lieu où l'on débat et soulève des questions et problèmes", un espace pour «la confrontation de différents points de vue, arguments et significations» (Scherer, 2011, p. 237) qui posent aux enseignants la question de leur rôle. Jusqu'où devraient-ils aller? Faut-il laisser-faire, modérer, participer? La réponse ne saurait être tranchée. Pensons aux «oppositions entre la contrainte et la liberté, l'effort et l'intérêt, [...] la culture générale et la spécialisation » (Reboul, 1989, p.13). Aucune de ces oppositions ne peut être surmontée par le triomphe de l'un des termes. Disons qu'au minimum, l'enseignant prépare l'espace «forum» en fonction de ses intentions, clarifie ses attentes et exploite quelques contributions (Verenikina, Jones et Delahunty, 2017). Quant au maximum, il n'existe pas, car l'enseignant n’a jamais fini d'améliorer son enseignement (Meirieu, 2014) : animer, répondre, modérer, relancer, questionner, comptabiliser des points en vue d'une évaluation finale...

Pour évaluer, Mazur (2014) a proposé un système de notation intéressant : 3 points pour une réponse parfaite ou presque; 2 points en cas de petites erreurs; 1 point en cas d'erreurs importantes et 0 quand la réponse est sans rapport. Ainsi, «la différence entre un 1 et un 2 est plus nette qu'entre un 6 et un 7 sur 10 » (p. 280). Ce barème conduirait à une notation cohérente peu contestable.

Notons que les utilisateurs du forum ont des attitudes diverses : certains en sont les habitants actifs ou silencieux; d'autres, de libres visiteurs, contributeurs précieux, mais de passage; restent quelques passants, sans intention ni intérêt (Scherer, 2011). Pour autant, celui qui s'y exprime n’en profite pas

2 - Formation et profession 29(2), 2021 
forcément davantage que celui qui s'y dérobe. La flânerie a l'étonnante vertu de plonger quelques privilégiés dans un interstice imprévu (Tesson, 2016), un terrier magique (Alice n’est jamais loin), si formateur, mais invariablement invisible à l'incessant parleur.

\section{Utilisez des outils collaboratifs.}

Ils se sont multipliés. Voici quelques fonctions non exhaustives que certains logiciels proposent :

- Partager des documents, les stocker, envoyer des fichiers lourds;

- Écrire en groupe, avec ou sans historique des contributions;

- Organiser le travail avec des tableaux et listes;

- Prendre des notes, créer des nuages de mots, des cartes mentales, des pense-bêtes;

- Créer un poster, créer une présentation;

- Produire des hypertextes en utilisant des wikis.

Ces logiciels peuvent susciter la curiosité, faciliter l'accès aux travaux des autres, réduire la paresse sociale en rendant visibles les contributions de chacun (Karau et Williams, 1993; Slavin, 1983), favoriser l'échange d'idées, enrichir les représentations...

\section{N'ayez pas peur de l'évaluation par les pairs}

La littérature ne s'attarde pas aux résistances que l'évaluation par les pairs peut faire naître; pourtant elles fleurissent : invocation d'un droit à être corrigé par l'enseignant, refus de corriger un camarade, stratégies de corrections accommodantes... L'enseignant déjouera ouvertement ces résistances, affinera une grille d'évaluation univoque à fournir aux étudiants et communiquera sur les bienfaits de l'activité. Car oui, les bienfaits sont nombreux : recul sur la matière par repérage de concepts sousjacents (Mazur, 2014), recul sur soi-même grâce à des exemples d'autres travaux (Brown et al., 2014), apprentissage vicariant (Bandura, 1977), engagement grâce à la précision des rétroactions d'étudiants (Galand et Vanlede, 2004), diminution de l'illusion de savoir (Brown et al., 2014). Et cerise sur le gâteau : l'enseignant y gagne en repérant des difficultés à travers les rétroactions d'étudiants ou en rendant possible des corrections individuelles en classes nombreuses...

\section{Interactions synchrones}

\section{Rendez les étudiants acteurs.}

Les étudiants ont besoin de place pour être actifs. Interpellez-les, proposez des échanges en sous-classe, utilisez des sondages ou le chat qui ont le mérite d'obtenir la contribution de tous simultanément, prévoyez des moments d'échange d'opinions (Levy, 2020). En cas de travail en sous-groupes ou de présentations devant la classe, il est fécond de remplir deux conditions. La première consiste à donner un but commun aux étudiants ; la seconde, à faire en sorte que les contributions individuelles de chacun restent saillantes (Slavin, 1983). Par exemple, il est productif de prévoir qu'une partie de l'évaluation d'un travail de groupe soit individuelle. 


\section{Donnez du rythme et du dynamisme}

La motivation des étudiants n'est pas innée, elle dépend de la relation, de l'interaction, de la situation, des désirs, des besoins (Perrenoud, 1994, p.162). Mobiliser est un défi posé à l'enseignant (Alexandre, 2017). Rythme et tempo sont importants. Le tempo, c'est la fréquence de la musique pédagogique. Si celle-ci est trop lente, l'ennui s'installe et le décrochage se fait rapidement. Le juste tempo est un milieu entre deux extrêmes, un équilibre précaire, un art. Une interprétation «très rapide et guillerette » du Clavier bien tempéré fit écrire à Hofstadter (2008) que les musiciens rataient l'essentiel, à vouloir réaliser un exercice de virtuosité «au lieu de transmettre un profond message sur la condition humaine » (p.460). Il s'agit d'une remarque à méditer pour toutes personnes faisant profession artistique (les enseignants en sont). Le rythme, quant à lui, intègre les notes et instruments, c'est-à-dire les activités et moyens, l'ensemble des composantes du cours. Les possibilités sont infinies : débuter par une accroche, interpeller ses étudiants, les challenger, se taire, varier les activités ou les outils...

\section{Conclusion}

À distance, les difficultés ressemblent à celles qui existent en présence, à une différence près : les adaptations de dernière minute sont plus embarrassantes. Enseigner consiste à relever un défi inépuisable : il y a toujours de quoi mieux faire, mieux organiser, mieux interagir, mieux expliquer. Concevoir l'enseignement à distance comme l'opportunité de se hasarder sur de nouvelles voies peut mener à des tentatives décevantes, certes, mais forcément enrichissantes.

\section{Accès au guide complet}

Baillifard, A., Carbonel, H., Favre, S. et Thurre-Millius, A., (2020). Favoriser les interactions synchrones et asynchrones en enseignement à distance. Digitalskills UniDistance.

https://digitalskills.unidistance.ch/wp-content/uploads/2020/11/Guide-Interactions-FR-2020.11.18.pdf

\section{Références bibliographiques}

Alexandre, D. (2017). Anthologie des textes clés en pédagogie. ESF.

Bandura, A. (1977). Self-efficacy : Toward a unifying theory of behavioral change. Psychological Review, 84(2), 191-215. https://doi.org/10.1037/0033-295X.84.2.191

Biggs, J. (1996). Enhancing teaching through constructive alignment. High Educ, 32, 347-364. https://doi.org/10.1007/BF00138871

Brown, P.C., Roediger, H. L. et McDaniel, M.A. (2014). Make it Stick: The science of successful learning. The Belknap Press of Harvard University Press.

Comeaux, P., Huber, R., Kasprzak, J. et Nixon, M.A. (1998). Collaborative Learning in Web-Based Instruction. US department of education. https://files.eric.ed.gov/fulltext/ED427693.pdf

Levy, D. (2020). Teaching effectively with Zoom: A practical guide to engage your students and help them learn. Dan Levy. ASIN : B08CBG9GRH

Galand, B. et Vanlede, M. (2004). Le sentiment d'efficacité personnelle dans l'apprentissage et la formation : quel rôle jouet-il? D'où vient-il? Comment intervenir ?. Savoirs : hors série, 5, 91-116. https://doi.org/10.3917/savo.hs01.0091 
Hameline D. (1979). Les objectifs pédagogiques. ESF.

Hofstadter, D. (2008). Je suis une boucle étrange. Dunod.

Houdé, O. (2017). Apprendre à résister. Le pommier.

Karau, S. J., \& Williams, K. D. (1993). Social loafing: A meta-analytic review and theoretical integration. Journal of Personality and Social Psychology, 65(4), 681-706. https://doi.org/10.1037/0022-3514.65.4.681

Korczak, J. (1920). Comment aimer un enfant suivi de Le droit de l'enfant au respect. (Z. Bobowicz, trad., 2006). Robert Laffont.

Mazur, E. (2014). Peer Interaction. Une méthode éprouvée d'enseignement interactif. Presses polytechniques et universitaires romandes.

Perrenoud, P. (1987). De l'école active à l'école interactive : un nouveau mythe? Dans CRESAS, On n'apprend pas tout seul! Interactions sociales et construction des connaissances (p.139-148). ESF.

Perrenoud, P. (1994). Métier d'élève et sens du travail scolaire. ESF.

Perrenoud, P. (1998). L'évaluation des élèves. De la fabrication de l'excellence à la régulation des apprentissages. Chapitre 8. De Boeck.

Meirieu, P. (2014). À l'école, offrir du temps pour la pensée. Esprit, 1 (1), 20-33. https://doi.org/10.3917/espri.1401.0020

Reboul, O. (1989). La philosophie de l'éducation. Que sais-je.

Scherer, S. (2011). Éducation à distance et interaction : L'importance des attitudes des enseignants et étudiants dans les forums et les wikis. Distances et savoirs, 2 (2), 235-248.

Slavin, R. E. (1983). When does cooperative learning increase student achievement? Psychological Bulletin, 94(3), 429-445. https://doi.org/10.1037/0033-2909.94.3.429

Tesson, S. (2016). Sur les chemins noirs. Gallimard.

Verenikina, I., Jones, P. et Delahunty, J. (2017). The Guide to Fostering Asynchronous OnlineDiscussion in Higher Education. Fostering OnLine Discussion, 1-26. https://doi.org/10.13140/RG.2.2.25787.26405

Webb, N. M. (1983). Predicting learning from student interaction: Defining the interaction variables. Educational Psychologist, 18(1), 33-41, https://doi.org/10.1080/00461528309529259

\section{Pour citer cet article}

Baillifard, A. (2021). 7 clefs pour des interactions pédagogiques porteuses d'apprentissages [Chronique]. Formation et profession, 29(2), 1-5. DOI http://dx.doi.org/10.18162/fp.2021.a231 